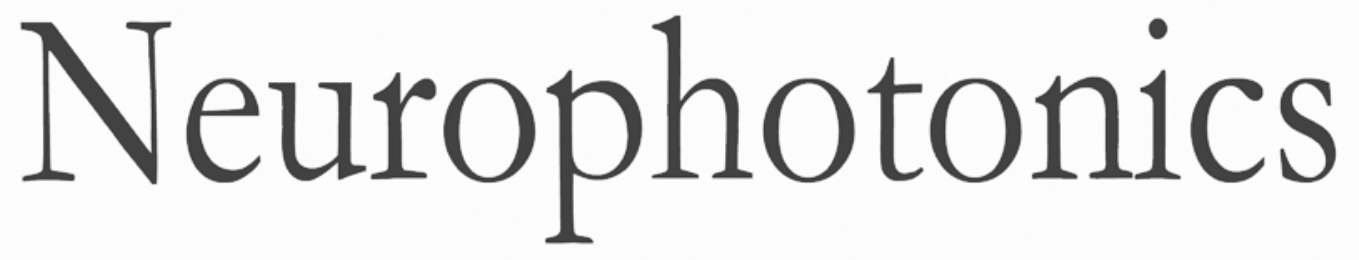

\title{
Intraoperative laser speckle contrast imaging with retrospective motion correction for quantitative assessment of cerebral blood flow
}

Lisa M. Richards

Erica L. Towle

Douglas J. Fox, Jr.

Andrew K. Dunn 


\title{
Intraoperative laser speckle contrast imaging with retrospective motion correction for quantitative assessment of cerebral blood flow
}

\author{
Lisa M. Richards, ${ }^{a}$ Erica L. Towle, ${ }^{a}$ Douglas J. Fox Jr., ${ }^{b}$ and Andrew K. Dunn ${ }^{a, *}$ \\ ${ }^{a}$ The University of Texas at Austin, Department of Biomedical Engineering, 107 W. Dean Keeton Street Stop C0800, Austin, \\ Texas 78712, United States

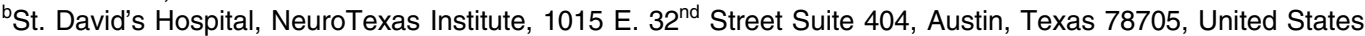

\begin{abstract}
Although multiple intraoperative cerebral blood flow (CBF) monitoring techniques are currently available, a quantitative method that allows for continuous monitoring and that can be easily integrated into the surgical workflow is still needed. Laser speckle contrast imaging (LSCI) is an optical imaging technique with a high spatiotemporal resolution that has been recently demonstrated as feasible and effective for intraoperative monitoring of CBF during neurosurgical procedures. This study demonstrates the impact of retrospective motion correction on the quantitative analysis of intraoperatively acquired LSCI images. LSCl images were acquired through a surgical microscope during brain tumor resection procedures from 10 patients under baseline conditions and after a cortical stimulation in three of those patients. The patient's electrocardiogram (ECG) was recorded during acquisition for postprocess correction of pulsatile artifacts. Automatic image registration was retrospectively performed to correct for tissue motion artifacts, and the performance of rigid and nonrigid transformations was compared. In baseline cases, the original images had $25 \% \pm 27 \%$ noise across 16 regions of interest (ROIs). ECG filtering moderately reduced the noise to $20 \% \pm 21 \%$, while image registration resulted in a further noise reduction of $15 \% \pm 4 \%$. Combined ECG filtering and image registration significantly reduced the noise to $6.2 \% \pm 2.6 \%(p<0.05)$. Using the combined motion correction, accuracy and sensitivity to small changes in CBF were improved in cortical stimulation cases. There was also excellent agreement between rigid and nonrigid registration methods (15/16 ROls with $<3 \%$ difference). Results from this study demonstrate the importance of motion correction for improved visualization of CBF changes in clinical LSCl images. $\odot$ The Authors. Published by SPIE under a Creative Commons Attribution 3.0 Unported License. Distribution or reproduction of this work in whole or in part requires full attribution of the original publication, including its DOI. [DOI: 10.1117/1.NPh.1.1.015006]
\end{abstract}

Keywords: cerebral blood flow; cerebral ischemia; flow measurement; image registration; intraoperative imaging; laser speckle contrast imaging.

Paper 14023R received Mar. 10, 2014; revised manuscript received Jun. 17, 2014; accepted for publication Jun. 24, 2014; published online Aug. 18, 2014.

\section{Introduction}

Because the brain relies on a constant supply of cerebral blood flow (CBF), any prolonged reduction of CBF can greatly increase the patient's risk for irreversible ischemic brain injury. By monitoring CBF during neurosurgery, surgeons can be promptly warned about a reduction in blood flow, allowing quick intervention to minimize the risks of postoperative neurologic deficits. Several intraoperative monitoring tools have been developed and evaluated in recent years, including digital subtraction angiography, ${ }^{1-3}$ electrophysiological monitoring, ${ }^{4-6}$ thermal diffusion flowmetry, ${ }^{7-9}$ microvascular Doppler sonography, ${ }^{10-12}$ and indocyanine green angiography. ${ }^{13-16}$ Recent reviews of these techniques during aneurysm clipping procedures concluded that none were completely reliable in all situations and that combining multiple techniques provided a more accurate picture of the hemodynamic state. ${ }^{17,18}$

Optical flow measurement techniques based on dynamic light scattering have the potential to improve many of the shortcomings of the currently available methods. Laser speckle contrast imaging (LSCI) is an optical imaging technique that provides high spatiotemporal resolution and quantitative

*Address all correspondence to: Andrew K. Dunn, E-mail: adunn@mail.utexas .edu measurements of blood flow. ${ }^{19,20}$ LSCI is a noncontact, noninvasive, full field, and real-time imaging technique that requires simple instrumentation, including a low-power laser diode, imaging optics, and a camera. Because a contrast agent is not required, imaging with LSCI can be repeatedly performed as needed or can be continuously performed to serve as a monitoring tool during intraoperative procedures.

LSCI has been widely studied in animal models, and has been recently investigated for clinical use during neurosurgery with promising results. Multiple studies have examined the utility of a commercial LSCI system during direct surgical revascularization, ${ }^{21,22}$ awake functional mapping, ${ }^{23}$ and cortical spreading depolarization after malignant stroke. ${ }^{24}$ All of these studies demonstrate the feasibility and usefulness of LSCI as an intraoperative tool for monitoring changes in CBF. However, these studies have not addressed correction for physiological motion artifacts, which have a direct influence on the motion-sensitive LSCI images. In this paper, we demonstrate the impact of retrospective motion compensation on the accuracy of quantitative blood flow analysis from LSCI images intraoperatively acquired during a pilot clinical study $(n=10)$. The goal of this analysis is to improve the visualization of small CBF changes in small tissue regions, which may otherwise be lost due to physiological noise. 


\section{Methods}

\subsection{Instrumentation}

An overall view of the hardware requirements for LSCI is shown in Fig. 1(a), with dashed lines indicating how each component is integrated into the clinical instrument. A schematic of the adapted microscope (Zeiss OPMI Pentero, Carl Zeiss Meditec Inc., Oberkochen, Germany) is shown in Fig. 1(b), with a corresponding photograph shown in Fig. 1(c). A laser diode ( $\lambda=660 \mathrm{~nm}, P=120 \mathrm{~mW}$, Thorlabs Inc., Newton, New Jersey) was incorporated into an add-on laser adapter (MM6 Micromanipulator, Carl Zeiss Meditec Inc.), which was attached to the bottom of the microscope head. The laser beam linearly travels through the adapter until it encounters a curved mirror, which directs the beam toward the cortex as shown in the laser adapter illustration of Fig. 1(a). The steering toggle on the laser adapter controls the angle of the mirror, which allows positioning of the beam on the tissue surface. The laser power was measured to be $28 \mathrm{~mW} / \mathrm{cm}^{2}$, which is far below the ANSI standard of $200 \mathrm{~mW} / \mathrm{cm}^{2}$ for maximum permissible exposure to a visible laser beam. ${ }^{25}$ A camera (Basler A602f, Basler Vision Technologies, Ahrensburg, Germany) was connected to one of the side viewing ports using a C-mount camera adapter (Carl Zeiss Meditec, Inc.). The camera used in this study had a sensor size of $656 \times 491$ pixels, a pixel size of $9.9 \mu \mathrm{m}$, and a frame rate of 100 images/second. This camera could be easily switched out for a camera model with a higher resolution or speed in future studies. The imaging optics built into the microscope were used to focus the camera and zoom onto an area of interest on the tissue surface. For this study, the microscope was set for maximum zoom and the camera field of view was $\sim 2 \times 1.5 \mathrm{~cm}$, corresponding to $\sim 3 \times$ demagnification relative to the camera sensor. The microscope's depth of field for the settings used during image acquisition was measured to be $\sim 5.7 \pm 0.6 \mathrm{~mm}$. The hardware modifications to the microscope were attached prior to the start of the surgery and did not interfere with sterile draping or with normal use of the microscope, as shown in Fig. 1(d).

\subsection{Intraoperative Procedure}

All experiments were performed during brain tumor resection surgeries at the NeuroTexas Institute in St. David's Hospital, Austin, TX. The clinical study was approved by the Institutional Review Boards of the University of Texas at Austin and St. David's Hospital and written informed consent was obtained for all patients. A summary of the patient details for the pilot clinical study is shown in Table 1. Immediately prior to LSCI imaging, the surgeon positioned the microscope over the cortical area of interest, adjusted the focus and zoom, and flushed the tissue with sterile saline to reduce specular reflections. LSCI images were recorded for $\sim 10-15$ min with a camera exposure time of $5.0 \mathrm{~ms}$. The camera exposure signal and the patient's electrocardiogram (ECG) waveform were simultaneously recorded during image acquisition for retrospective cardiac cycle motion compensation. Baseline blood flow images were recorded for all patients, and in three cases, images were acquired before and after cortical stimulation with either bipolar cautery or the Ojemann cortical stimulator.

\subsection{Image Analysis}

The raw images recorded by the camera were converted into speckle contrast images using Eq. (1),

$$
K=\frac{\sigma_{s}}{\langle I\rangle}
$$

where the spatial speckle contrast, $K$, is defined as the ratio of the standard deviation, $\sigma_{s}$, to the mean intensity of pixels, $\langle I\rangle$,

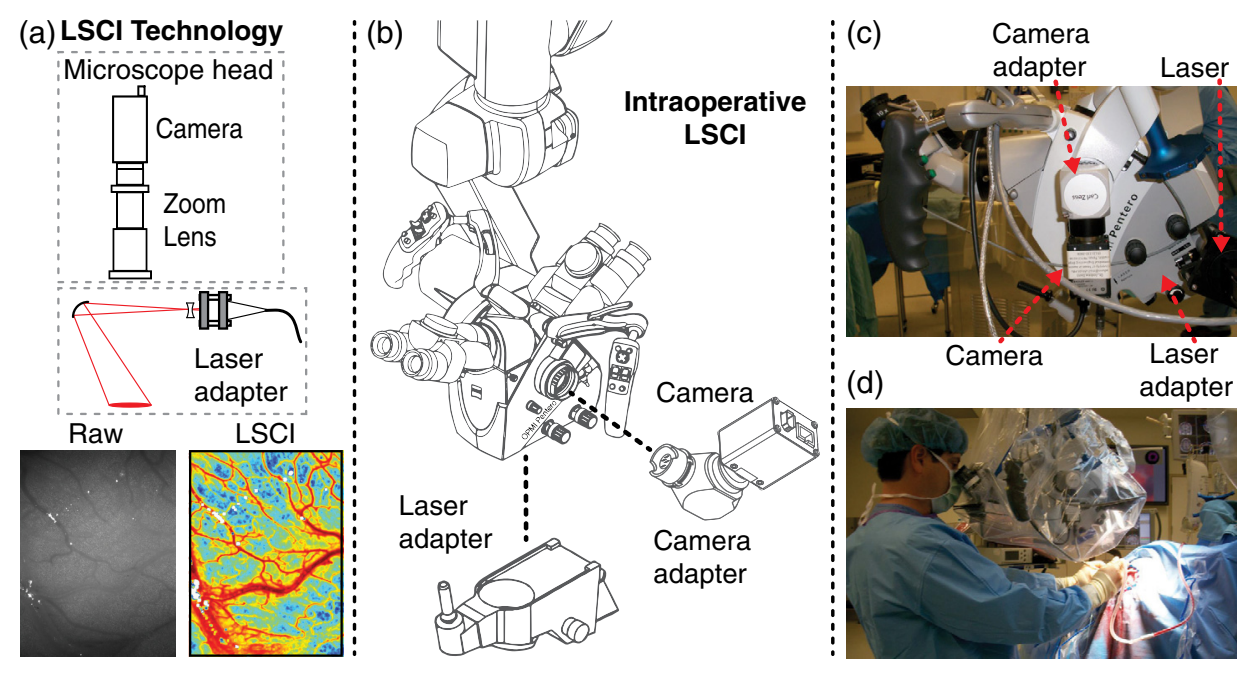

Fig. 1 Zeiss OPMI Pentero neurosurgical microscope adapted to measure the cerebral blood flow intraoperatively using LSCl. (a) A schematic showing the hardware requirements for $\mathrm{LSCl}$ and how these are adapted for intraoperative use. The imaging optics are built-in to the microscope head, the camera is attached to a side viewing port, and the laser illumination is built in to an add-on laser adapter. Representative images from a clinical case illustrate the raw image recorded by the camera (Raw) and the corresponding speckle contrast image (LSCl). (b) A schematic of the intraoperative instrumentation shows the add-on attachments for LSCl, with drawings adapted from the Zeiss OPMI Pentero Manual Issue 9.3. (c) Photograph of the intraoperative instrumentation with all add-on components labeled. (d) The LSCl adapted microscope is covered in a sterile drape and used to assist with the tumor resection procedure without any interference from the additional hardware. 
Table 1 Clinical patient overview for the pilot study. All patients were undergoing brain tumor resection procedures and were imaged either before or after the resection at the discretion of the surgeon. ${ }^{a}$

Patient Gender Age First brain surgery? Previous treatment? Craniotomy position LSCl versus resection? Baseline versus stimulation?

\begin{tabular}{|c|c|c|c|c|c|c|c|}
\hline 1 & $\mathrm{M}$ & 52 & $Y$ & $\mathrm{~N} / \mathrm{A}$ & R Temporal & Before & Baseline \\
\hline 2 & M & 33 & $\mathrm{~N}$ & Chemo & R Frontal & After & Baseline \\
\hline 3 & $\mathrm{~F}$ & 25 & $\mathrm{~N}$ & Cranial XRT, chemo & R Fronto-temporal & Before & Baseline \\
\hline 4 & $\mathrm{~F}$ & 57 & $\mathrm{Y}$ & $\mathrm{N} / \mathrm{A}$ & L Sphenoid wing & After & Bipolar cautery \\
\hline 5 & $\mathrm{~F}$ & 62 & $\mathrm{Y}$ & $\mathrm{N} / \mathrm{A}$ & L Frontal above orbit & After & Baseline \\
\hline 6 & M & 64 & $\mathrm{Y}$ & $\mathrm{N} / \mathrm{A}$ & L Posterior Frontal & After & Ojemann cortical stimulator \\
\hline 7 & $\mathrm{M}$ & 57 & $Y$ & $\mathrm{~N} / \mathrm{A}$ & R Frontal & After & Baseline \\
\hline 8 & M & 70 & $\mathrm{~N}$ & $\mathrm{~N} / \mathrm{A}$ & R Parietal & After & Baseline \\
\hline 9 & $\mathrm{~F}$ & 75 & $\mathrm{Y}$ & $\mathrm{N} / \mathrm{A}$ & L Occipital & After & Baseline \\
\hline 10 & $\mathrm{~F}$ & 42 & $Y$ & Cranial XRT & Bifrontal, falcine based & After & Bipolar cautery \\
\hline
\end{tabular}

${ }^{\mathrm{a} X R T}=$ radiation therapy

in a $7 \times 7$ pixel region of the image. ${ }^{26}$ Sliding this window throughout the raw image creates the full-field speckle contrast image. The spatial speckle contrast analysis quantifies the local spatial variance, or blurring, of the speckle pattern that results from the decorrelation of coherent light in the presence of blood flow. Representative raw and speckle contrast images from a clinical case are shown in Fig. 1(a). By incorporating rapid processing techniques ${ }^{27}$ into the custom software used for image acquisition, the speckle contrast images were computed and displayed in real time for live visualization of CBF during the procedure. All images in the paper are displayed as speckle contrast images, since these represent an instantaneous map of blood flow.

The remaining image analysis was performed in postprocessing after the completion of each clinical case. To provide a more quantitative measure of blood flow, the speckle contrast images were converted to correlation time, $\tau_{c}$, using Eq. (2),

$$
K\left(T, \tau_{c}\right)=\left(\beta \frac{e^{-2 x}-1+2 x}{2 x^{2}}\right)^{1 / 2},
$$

where $x=T / \tau_{c}, T$ is the exposure time of the camera, $\tau_{c}$ is the correlation time, and $\beta$ is an instrumentation factor that depends on polarization, coherence effects, and speckle averaging. ${ }^{28}$ For this study, $\beta$ was assumed to be equal to 1 , which affects the absolute value of $\tau_{c}$ but does not affect computations of relative changes in blood flow. CBF was calculated as the flow relative to a baseline value, which is the ratio of an average baseline $\tau_{c}$ value and the $\tau_{c}$ values at all remaining time points. ${ }^{29,30}$ The baseline was defined as the average $\tau_{c}$ over $\sim 5 \mathrm{~s}$ at the start of acquisition for each region of interest (ROI) analyzed. For all plots in the paper, the deviation in CBF from the baseline is shown, $\% \Delta$, which makes it easy to visualize the change in flow relative to the baseline value.

\subsection{Cardiac Filtering}

To account for blood flow changes that occur during the cardiac cycle, an ad hoc ECG filter ${ }^{31-33}$ was implemented to reduce fluctuations in recorded $\tau_{c}$ values. Briefly, the actual time of each image was determined from the recorded camera exposure signal and the actual time of each heartbeat was determined from the ECG waveform. A "normalized time" was assigned to each image frame based on the relative time of the image between adjacent $R$ peaks in the cardiac cycle. The measured $\tau_{c}$ was plotted against the normalized time for the first 25 heartbeats and a large-window moving average filter was used to compute the ad hoc filter function. This filter was applied to the entire data set by subtracting the filter value for the corresponding normalized time and adding back the median $\tau_{c}$ of the filter function. A small-window moving average filter (width $=0.15 \mathrm{~s}$ ) was used to reduce the noise in the filtered $\tau_{c}$ result. This cardiac filtering helps to reduce the variation in $\tau_{c}$ due to the cardiac cycle while retaining inherent variability due to physiological changes in flow. ${ }^{31}$

\subsection{Image Registration}

Image registration was performed on the speckle contrast images using Elastix. ${ }^{34}$ This program performs an automatic intensity-based image registration by adjusting the position of a moving image to match a fixed image using the transformation type, similarity measure, and optimization procedure specified by the user. The similarity measure was set to mutual information and the optimization procedure was set to adaptive stochastic gradient descent based on the registration performance. The transformation type was set to either translation transform for rigid registration or B-spline transform for nonrigid registration, and a comparison between the two transforms was performed. The $x$ - and $y$-offsets needed for mapping each moving image back to the fixed image were output from Elastix in the translation transform parameter file. An overall total displacement was calculated for each registered image to assess the spatial shift between the original and registered images. 


\section{Results}

\subsection{Clinical Study Overview}

The speckle contrast images serve as blood flow maps, where red regions correspond to faster flow and blue regions correspond to slower flow. Figure 2 shows the camera field of view (FOV) recorded by the Zeiss built-in color camera under xenon lamp illumination along with corresponding registered speckle contrast images for two patients. The speckle contrast images were also thresholded to display only speckle contrast values less than $40 \%$ of the maximum (fastest flows) and were overlaid on top of the color photographs. These image sets show excellent alignment of the anatomical vasculature and the blood flow maps seen in the LSCI images, confirming the performance of the microscope-integrated LSCI system. The overlay image also highlights a major benefit of integrating LSCI into the surgical microscope: LSCI can provide blood flow maps for the tissue region in the microscope's FOV with the spatial resolution of the microscope.

\subsection{Retrospective Motion Correction}

The result of applying the ECG filtering procedure on original and registered image sets is shown for a single patient in Fig. 3, where the measured $\tau_{c}$ has been converted into CBF $\% \Delta$ for ease of interpretation. Figure 3 shows the alignment of the minimum CBF with the ECG R-peak [Fig. 3(b)], the ECG filter shape generated from 25 heartbeats [Figs. 3(c) and 3(e)] and the filtered results [Figs. 3(d) and 3(f)]. Cardiac artifacts are clearly present both before and after registration from the measured CBF in blue. The filter shape is altered after registration, as tissue movement artifacts that result in sampling different tissue areas manifest as CBF changes. The filtered output reduces beat-to-beat variability from $12 \%$ to $3.0 \%$ [standard deviation of blue versus black curves in Fig. 3(f)], which is consistent with previously published results ${ }^{31}$ and allows the possibility of visualizing small changes in CBF. All plots in subsequent figures have been ECG filtered and display the equivalent of the black curves from Figs. 3(d) and 3(f).

Translation rigid registration was performed for all image data because of the decreased complexity, reduced computation time, and preservation of image integrity. A comparison between the original and registered image sets is shown for three baseline cases in Fig. 4. These plots illustrate greater stability and reduced fluctuations in the ROI time courses after registration, especially for the vessel ROIs. This confirms that the CBF changes in the original time courses were solely due to tissue motion, causing vessel ROIs to sample the parenchyma, and vice versa. Compared to the dramatic improvement for vessel ROIs, registered parenchyma ROIs are visually similar to their originals, especially those placed farther from nearby vessels. A movie showing the original and translation-registered image sets for a single baseline case is included to demonstrate the effectiveness of image registration for correcting tissue motion artifacts in LSCI images (Video 1, left and center panels).

As seen in the left frame of Video 1 and in the original plots from Fig. 4, the lateral displacement caused by tissue motion can be a significant source of noise, especially for vessel tissue regions. This displacement was quantified as the total spatial shift of each registered image relative to the fixed image at the start of acquisition. Across seven cases, the mean lateral displacement ranged from $0.12 \pm 0.078$ to $0.64 \pm 0.15 \mathrm{~mm}$, and the maximum displacement ranged from 0.41 to $1.9 \mathrm{~mm}$. To illustrate how tissue motion affects the measured $\mathrm{CBF}$ changes, the relationship between the change in $\tau_{c}$ and displacement is illustrated in Fig. 5 for two cases corresponding to Figs. 4(b) and 4(e). The displacement is co-localized in time with the change in $\tau_{c}$, showing excellent correspondence between the magnitude of the spatial shift and the change in flow measured by LSCI. Pearson's correlation coefficient indicates a positive correlation between the displacement and change in flow, with $r \geq 0.9$ for two of the ROIs.
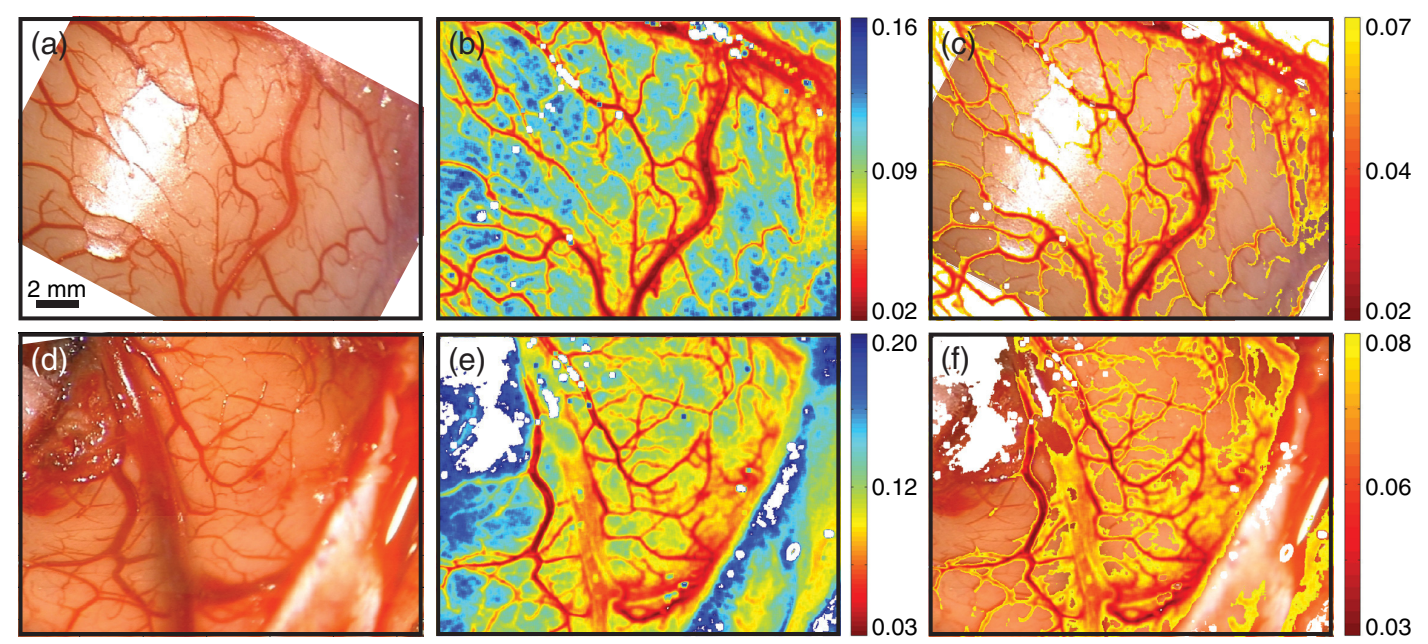

Fig. 2 Comparison between color photographs taken under xenon lamp illumination using the built-in Zeiss color camera (a,d) and corresponding LSCI images (b, e) from patients 7 and 9, respectively. The color photographs were registered to match the orientation of the speckle contrast images for ease of comparison. The speckle contrast images are averaged over 30 frames with a field of view of $\sim 2 \times 1.5 \mathrm{~cm}$. The scale bar in (a) applies to all images. The color map displays high flows in red (low speckle contrast) and low flows in blue (high speckle contrast), and the bright white areas are regions of specular reflection. The LSCl images were thresholded to display the speckle contrast values less than $40 \%$ of the maximum and are overlaid on top of the color photographs (c, $f$ ). 
(a)

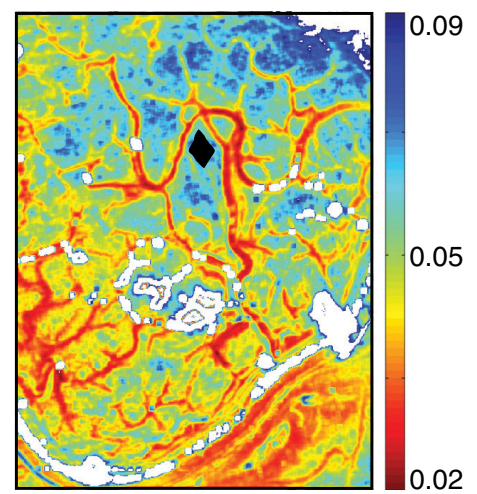

(c)
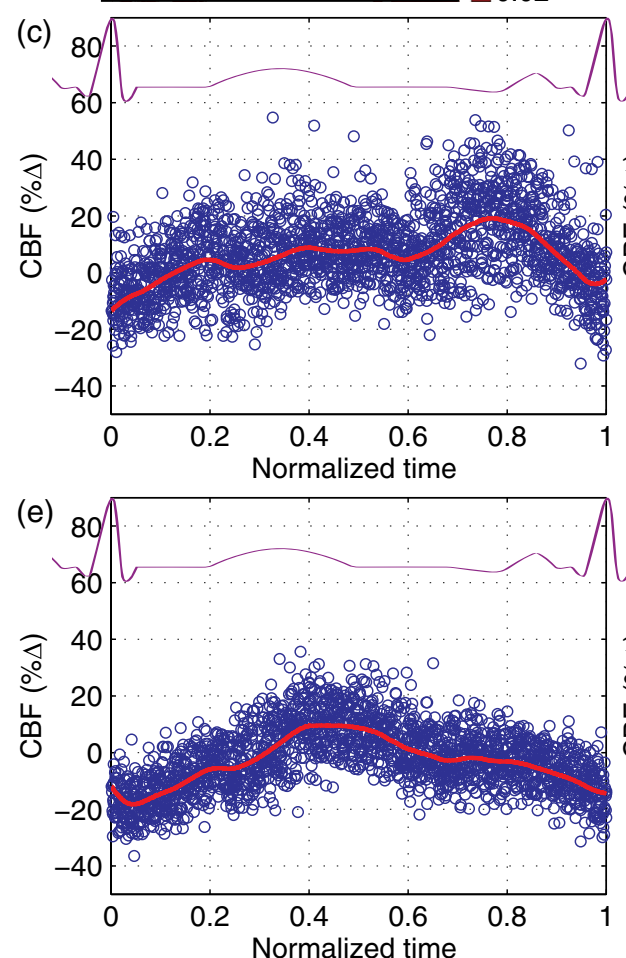

$\longrightarrow=$ Experimental Measurements
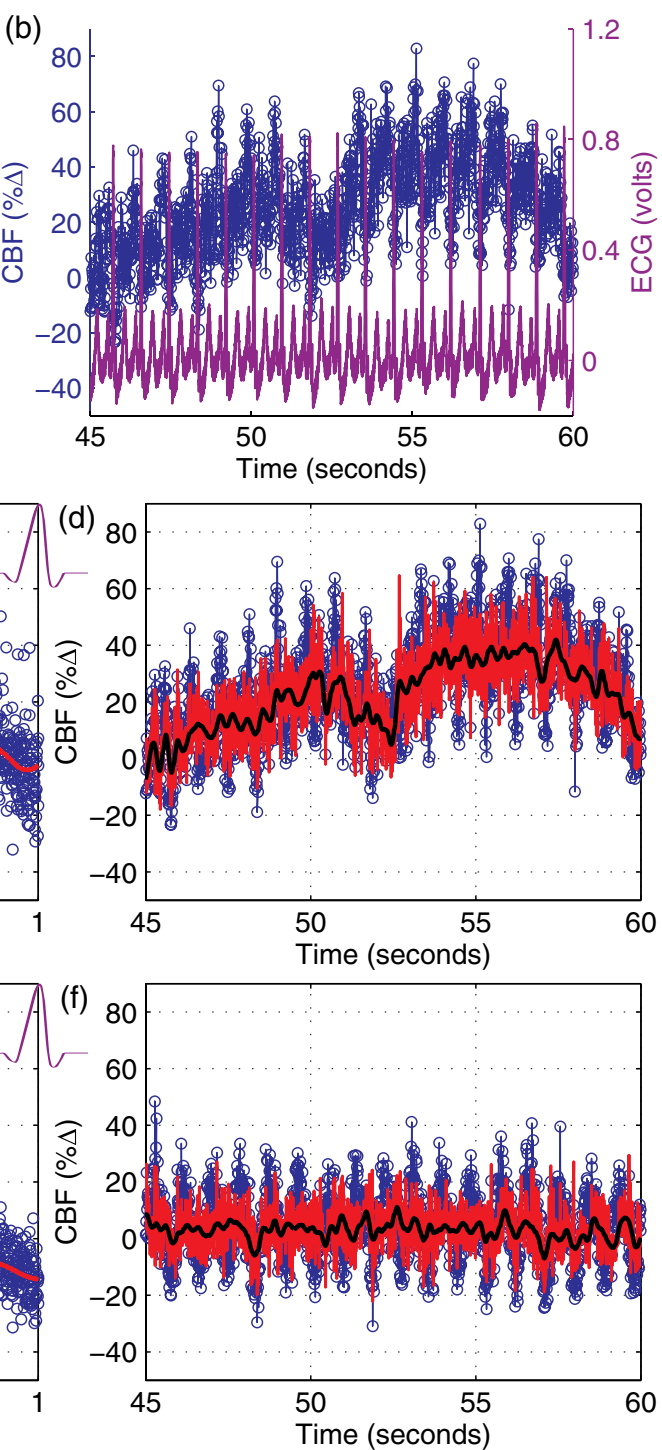

$-=$ ECG Signal
$-=E C G+$ Moving Average Filter

Fig. 3 The result of applying the ECG filtering process used to remove the pulsatile motion artifacts before and after translation-based image registration for patient 4 . (a) The speckle contrast image is averaged over 30 frames with a field of view of $\sim 2 \times 1.5 \mathrm{~cm}$ [same scale bar from Fig. 2(a)]. The $\mathrm{ROI}$ is shown in black, and the color map displays high flows in red (low speckle contrast) and low flows in blue (high speckle contrast). (b) The average CBF percent change $(\% \Delta)$ computed from the ROI is co-localized in time with the ECG waveform recorded for the patient, showing alignment of minimum CBF with the ECG R-peak. The CBF $\% \Delta$ is calculated using an average of $\sim 5 \mathrm{~s}$ of baseline flow at the start of acquisition. (c) The ad hoc ECG filter shape generated from 25 heartbeats from the original dataset. (d) The ECG filtered output is shown in red and the result after applying a small-window moving average filter to the filtered output is shown in black for the original dataset. (e) The ad hoc ECG filter shape generated from $\sim 25$ heartbeats from the translation-based registered dataset. (f) The ECG filtered results with and without the small-window moving average filter from the translation-based registered data set. The black curve from (d) or (f) will be displayed in future plots.

Quantitative analysis confirms that both vessel and parenchyma regions had a statistically significant noise reduction after ECG filtering plus registration (Fig. 6). Noise was quantified as the standard deviation of the $\mathrm{CBF} \%$ over the recorded time course for each ROI analyzed. Noise levels were compared for the original recorded images versus ECG filtering alone, image registration alone, and ECG filtering plus image registration for 16 ROIs across four baseline cases. ECG filtering alone as well as combined with image registration are the two scenarios highlighted in the plots from Fig. 4. As shown in Fig. 6, ECG filtering alone causes a statistically significant noise reduction in the parenchyma regions $(15 \% \pm 3 \%$ to $8.3 \% \pm 2.7 \%$, $p<10^{-5}$ ). Registration alone causes a statistically significant noise reduction in the vessel regions relative to ECG filtering alone $(38 \% \pm 25 \%$ to $16 \% \pm 5.8 \%, p<0.05)$, as well as a large reduction relative to the vessel noise in the original set. However, the most dramatic noise reduction is seen after combining both ECG filtering and image registration, which had 

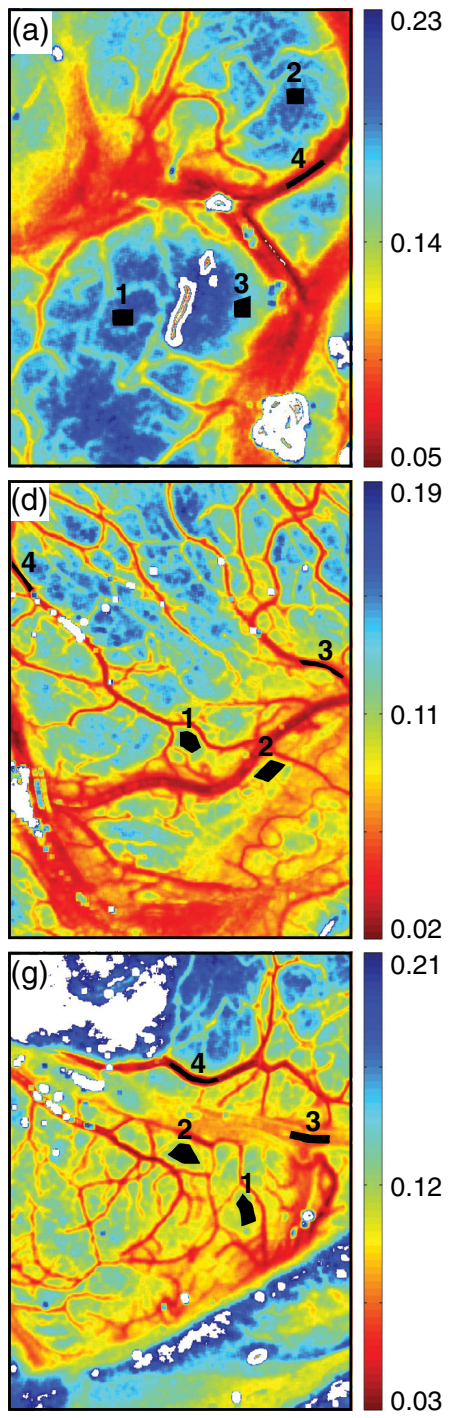
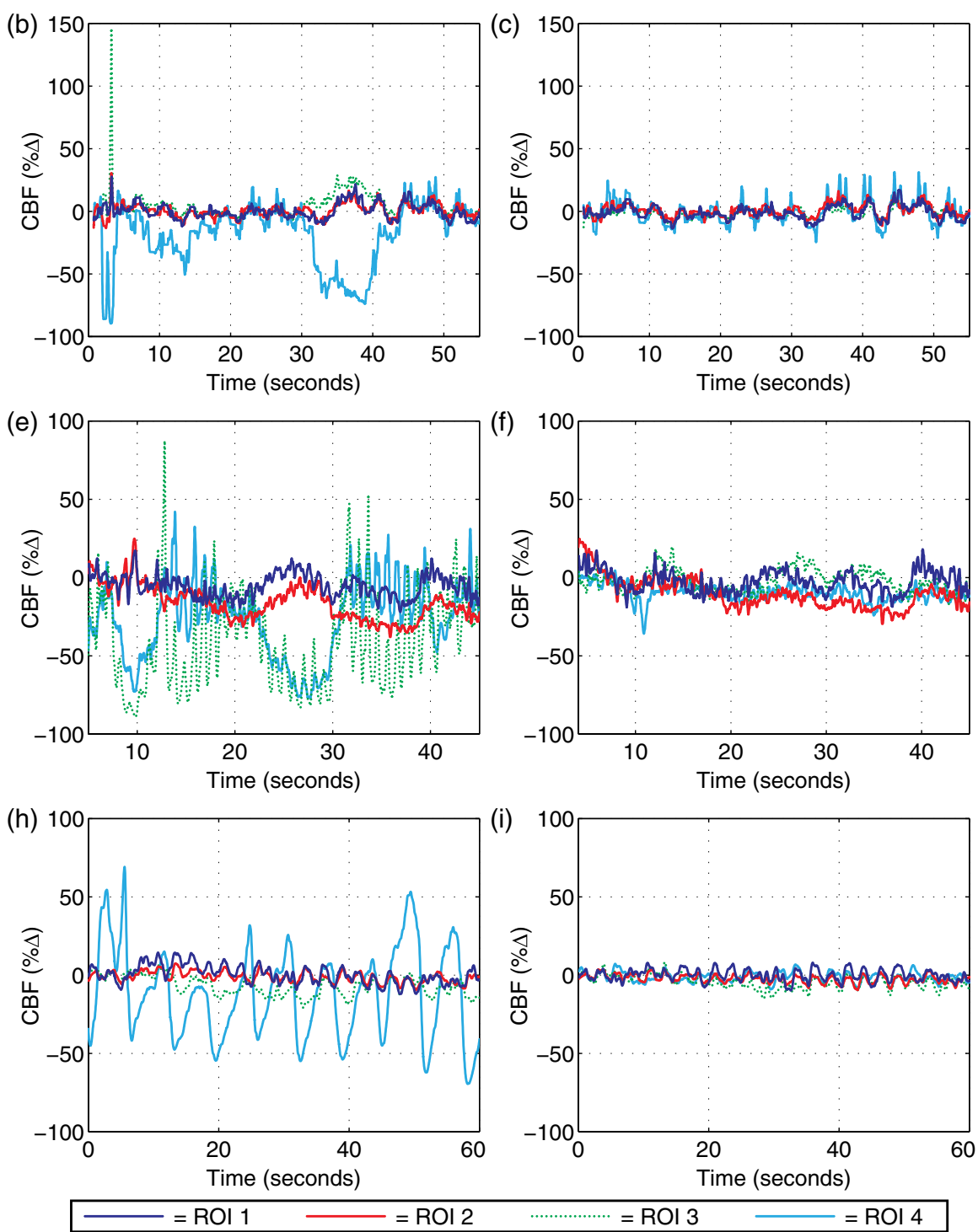

Fig. $4 \mathrm{~A}$ comparison between the time courses of ROls before and after translation-based image registration for three baseline cases, patients 1,7 , and 9 for parts (a)-(c), (d)-(f), and (g)-(i), respectively. The images on the left $(a, d, g)$ display the speckle contrast image at the start of image acquisition averaged over 30 frames with a field of view of $\sim 2 \times 1.5 \mathrm{~cm}$ [same scale bar from Fig. 2(a)]. The color map displays high flows in red (low speckle contrast) and low flows in blue (high speckle contrast) with four numbered ROls overlaid in black where analysis is taking place. The middle column of plots $(b, e, h)$ displays the time course of the original recorded CBF percent change $(\% \Delta)$ during the course of the intraoperative acquisition. The right column of plots $(\mathrm{c}, \mathrm{f}, \mathrm{i})$ displays the time course of the $\mathrm{CBF} \% \Delta$ after translation-based image registration of the image set. The $\mathrm{CBF} \% \Delta$ is calculated using an average of $\sim 5 \mathrm{~s}$ of baseline flow at the start of acquisition. Plots for the same patient were plotted with the same axes for easier comparison before and after registration.

a statistically significant reduction for all tissue regions. Combined ECG filtering and image registration significantly reduced the noise across all ROIs to $6.2 \% \pm 2.6 \%$. This was statistically significant compared to the original set $(25 \% \pm 27 \%, p<0.05)$, ECG filtering alone $(20 \% \pm 21 \%$, $p<0.05)$, and image registration alone $\left(15 \% \pm 4 \%, p<10^{-5}\right)$.

For the three cortical stimulation cases, a comparison between the original and registered images is shown in Fig. 7. Similar to the original baseline cases, large-scale fluctuations resulting from tissue motion make it difficult to accurately quantify and interpret the flow dynamics, especially in Figs. 7(b) and 7(e). After registration, the noise is largely suppressed and clear trends in $\mathrm{CBF}$ change are easily visualized in each case. Quantification of the flow change confirms that region 5 experienced the smallest change from baseline in all cases with a flow reduction of $24 \%$, flow increase of $31 \%$, and flow decrease of $46 \%$ for Figs. 7(c), 7(f), and 7(i), respectively. This improved sensitivity to small changes in flow increases LSCI's utility as a CBF monitoring tool. Removing the tissue motion artifact also increases the accuracy of the results and patient safety, as the original region 5 time courses misleadingly suggested larger flow reductions in Figs. 7(b) and 7(e) that may have prompted unnecessary surgical action. 


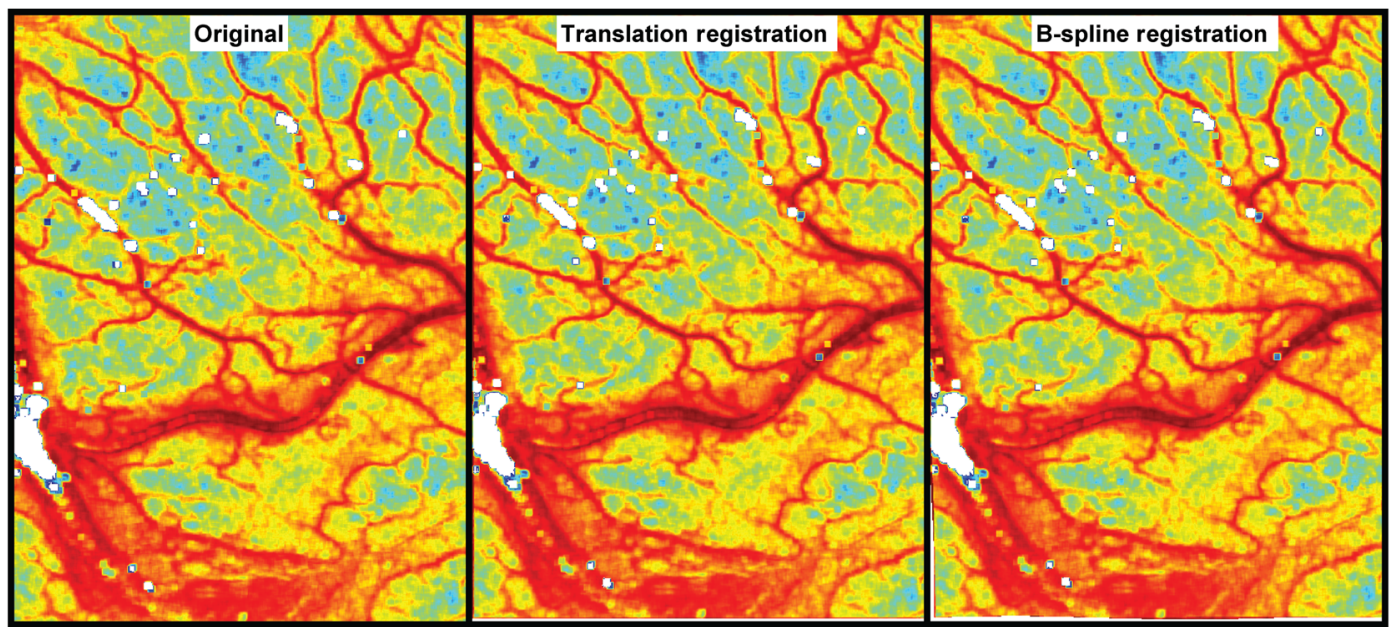

Video 1 This video demonstrates the impact of both translation and B-spline-based image registration on a LSCI data set for monitoring CBF. The left frame shows the original image set from baseline case 7 , the middle frame shows the translation-registered image set, and the right frame shows the B-spline registered image set, all with five-frame averaging. The location of the vasculature is stable after translation registration, which allows accurate quantitative monitoring of $\mathrm{CBF}$ changes in different tissue regions over time. B-spline registration provides both lateral image correction given by translation registration and axial correction for tissue pulsation and other nonrigid deformations (MOV, 12.8 MB) [URL: http://dx.doi.org/10.1117/1.NPh.1.1.015006.1].

Since brain tissue may deform after skull removal, a nonrigid transform may be required to account for tissue deformation. Because of increased computational costs, we performed B-spline nonrigid registration on a subset of the pilot study data from four baseline patients for comparison to the translation transform, with results shown for a single patient in Fig. 8. Similar to Fig. 4, both registration methods greatly reduce the fluctuations and improve stability, with the most significant impact on the vessel ROI and visible improvement on parenchyma regions placed near vessels. When Figs. 8(c) and 8(d) are directly compared, the time courses are visually similar between the two registration methods. Video 1 (middle and right panels) includes a comparison of translation and B-spline registered image sets for a single baseline case to demonstrate
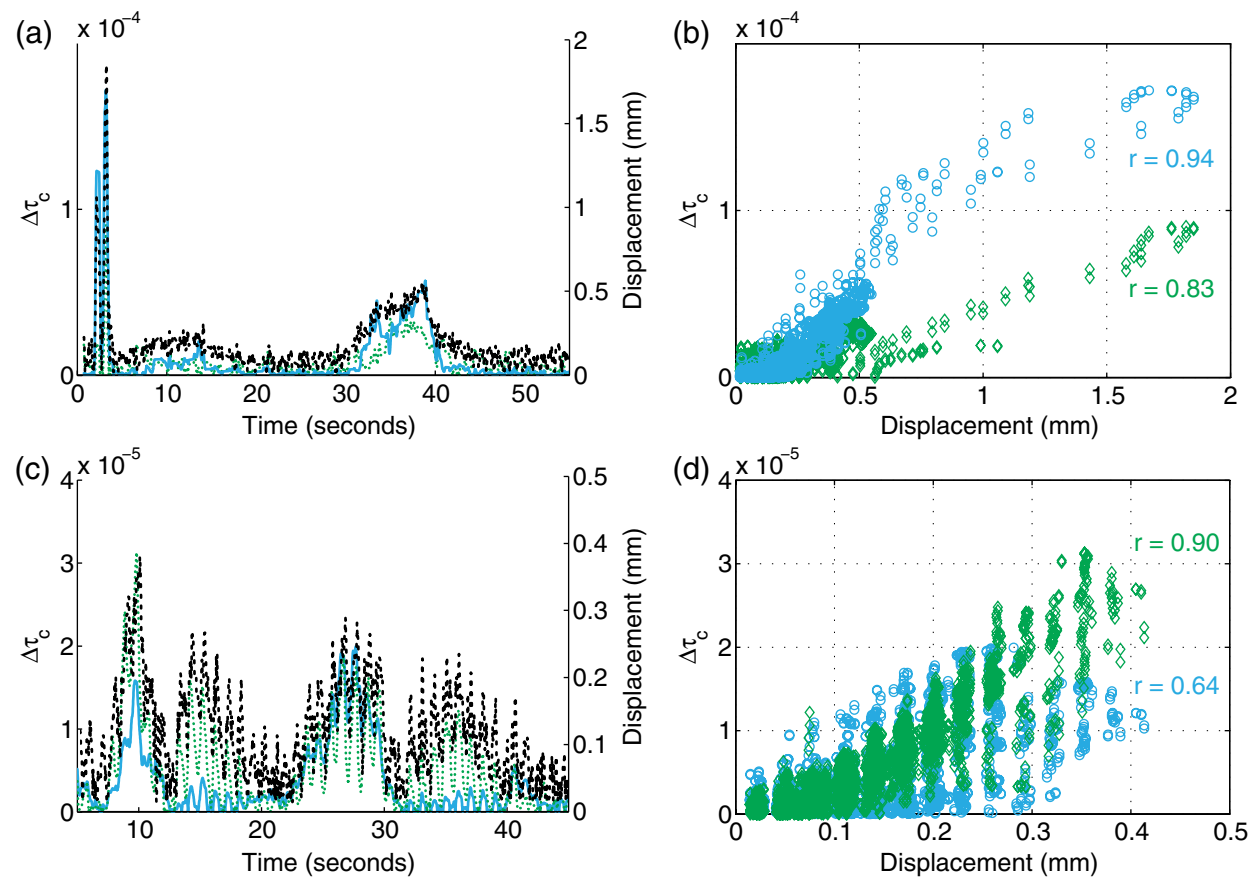

Fig. 5 (a, c) The displacement is co-localized in time with the change in correlation time $\left(\Delta \tau_{c}\right)$ for two ROls in Figs. 4(b) and 4(e), respectively. Two ROls are shown for each case to improve the visibility on the plots, and the colors correspond to those in Fig. 4. The black dashed line corresponds to the displacement. (b, d) Correlation between the displacement and $\Delta \tau_{c}$ for $(a, c)$, respectively, with the Pearson's correlation coefficient listed for each ROI. 


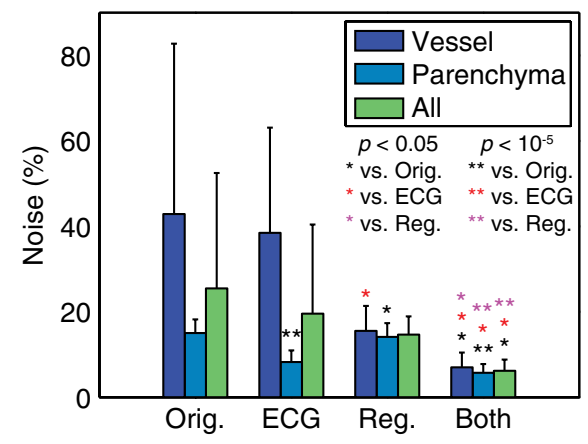

Fig. 6 Quantification of noise (standard deviation of CBF \%) before and after both ECG filtering and image registration across ROI groups from baseline cases $1,5,7$, and 9 . Statistical significance is calculated using a right-tailed paired $t$-test. Sample sizes are $N=6$ for vessel ROls, $N=10$ for parenchyma ROls, and $N=16$ for all ROls. Orig. = original data set; ECG = ECG filtering only; Reg. = image registration only; and Both = ECG filtering plus image registration.

the effectiveness of nonrigid image registration for correcting axial tissue deformation artifacts in the LSCI image set.

Quantitatively, the average percent difference between rigid and nonrigid registration methods is displayed in Table 2 for the ROIs shown in Figs. 4(a), 4(d), 4(g), and 8(a). Although Bspline registration is effective for removing residual nonrigid tissue deformation artifacts that occur in the axial direction, the difference compared to translation registration was minimal, with $<1 \%$ difference for 11/16 ROIs and $<3 \%$ difference for 15/16 ROIs. Region 4 in Fig. 8(a) had the largest discrepancy with a $7 \%$ difference. However, the improvement of B-spline registration was only moderate relative to translation registration when viewing region 4 over time. Overall, the lower complexity translation registration had excellent agreement with B-spline registration and the improved motion correction indicated by the small percent differences would not change the course of treatment.

\section{Discussion}

Many groups have demonstrated the feasibility of using LSCI as an intraoperative monitoring tool. ${ }^{21-24,31-33}$ As LSCI gains popularity and clinical investigations increase, it is important to assess the impact of physiological motion and investigate methods to compensate for these artifacts. Because LSCI is inherently sensitive to motion, the recorded images are impacted by both pulsatile flow and tissue deformation from the cardiac cycle and respiration. This paper utilized the ECG filtering method ${ }^{31}$ to reduce the beat-to-beat variability from $12 \%$ to $3 \%$, making it possible to visualize small changes in the flow. LSCI has the potential to be used as a monitoring tool during functional activation procedures such as awake cranial mapping, where the change in blood flow may be as low as $5 \%$ to $10 \% .{ }^{35}$ Pulsatile changes in flow can mask these small changes, making ECG filtering crucial for increasing applicability of the technique to a wide range of neurosurgical procedures. Although other techniques could be used for cardiac filtering, there are disadvantages associated with each. A large-window moving average filter alone (width $=0.5$ ) can be used to reduce the beat-tobeat variability from $12 \%$ to $2.8 \%$; however, the ability to observe transient physiological blood flow changes is diminished. ${ }^{31}$ Alternatively, the image acquisition can be synchronized with the cardiac cycle to minimize both the cardiac shift and the $\mathrm{CBF}$ measurement error. However, gating the signal during acquisition will reduce the effective frame rate by $24 \%$ to retain data $\pm 10 \%$ from baseline and by $63 \%$ to retain data $\pm 5 \%$ from baseline. This will reduce both the continuity of the data and the ability to perform frame averaging.

Image registration was added to improve the ability to track tissue ROIs accurately over time with significant noise reduction. The image registration method used in this study employs a similar procedure to a reported rodent study, ${ }^{36}$ which found significant spatial de-noising after registration. The flow dynamics are easier to interpret and quantify compared to the original time courses, especially for vessel regions and parenchyma regions placed near a vessel. The lateral displacement caused by tissue motion was quantified, ranging from a maximum of 0.41 to $1.91 \mathrm{~mm}$ across cases with excellent correspondence to the change in flow measured by LSCI. Noise reduction was quantified across 16 ROIs of different tissue types, with ECG filtering alone leading to a significant noise reduction in the parenchyma regions and image registration alone leading to the largest noise reduction in the vessel regions. These motion correction techniques could be individually used if the imaging application focused on only certain tissue types. By combining ECG filtering and image registration, noise levels were significantly reduced across all ROIs to $6.2 \% \pm 2.6 \%$, which makes it possible to visualize small changes in the flow from any tissue region. The motion correction techniques improved visualization of stable CBF levels during baseline cases and relative changes in blood flow after cortical stimulation, with a measured flow reduction as small as $24 \%$. Although LSCI can only provide relative flow information, the ability to identify change in blood flow is clinically most important. LSCI provides both the relative magnitude and the temporal dynamics of the blood flow change with the help of motion correction, which is the information the surgeon needs for clinical decision making. Although translation-based image registration is computationally intensive, combined ECG filtering and image registration provides the highest level of noise reduction, allowing visualization of blood flow changes. The results from this study suggest that intraoperative implementation of motion compensation techniques will increase the value and efficacy of LSCI as a CBF monitoring tool.

Correction for axial displacement was also examined using B-spline nonrigid registration. ROI analysis for B-spline registration was comparable to translation registration $(<3 \%$ difference across 15/16 ROIs), confirming that translation registration is sufficient for motion correction to reduce the computational costs. Although depth correction was unnecessary for accurate quantitative analysis, it is important that the images remain in focus throughout the full range of axial displacement observed to ensure accurate CBF estimates from LSCI. Given the microscope's measured depth of field, the maximum axial range must be $\leq 5.1 \mathrm{~mm}$ to maintain the focus during acquisition. As seen easily in Video 1, the pulsatile motion of the brain during the cardiac cycle results in axial motion. The axial displacement of the brain during the cardiac cycle has been measured to be up to $2 \mathrm{~mm},{ }^{37}$ which is still well within the microscope's depth of field. The curvature of the brain can also affect the axial range of the image. For the three-dimensional cortical surface of the brain, the large-scale curvature can be estimated using the mean geodesic of the brain, which corresponds to a $64-\mathrm{mm}$ radius osculating sphere. ${ }^{38}$ This corresponds to an axial range of $1.2 \mathrm{~mm}$ for the $\sim 2 \times 1.5 \mathrm{~cm}$ FOV, which is still well within the depth of field. The local curvature of the cortex 

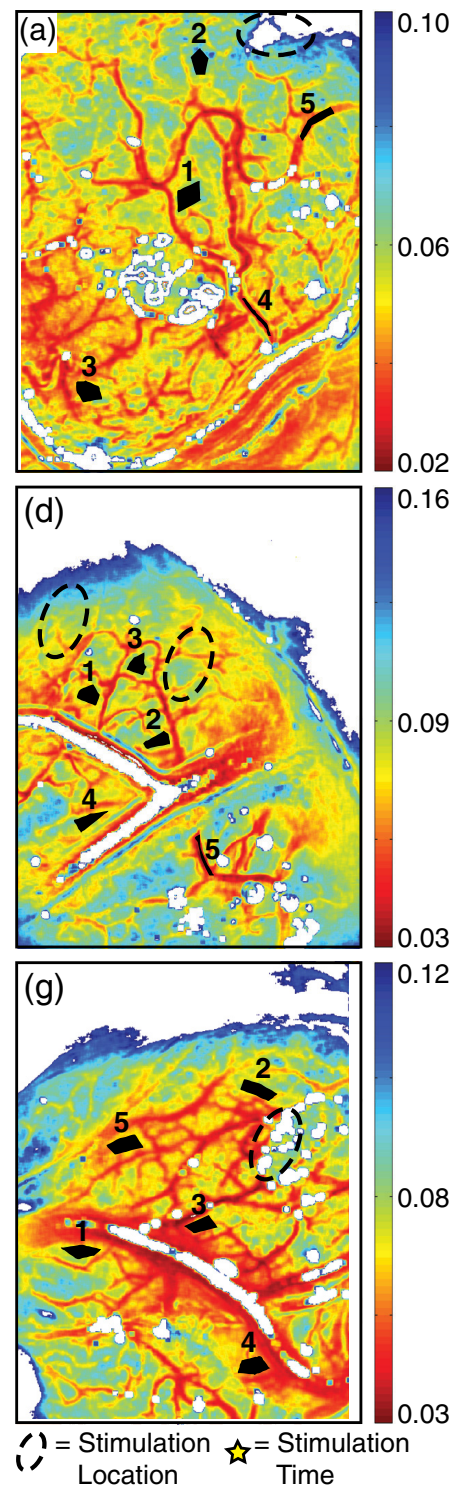
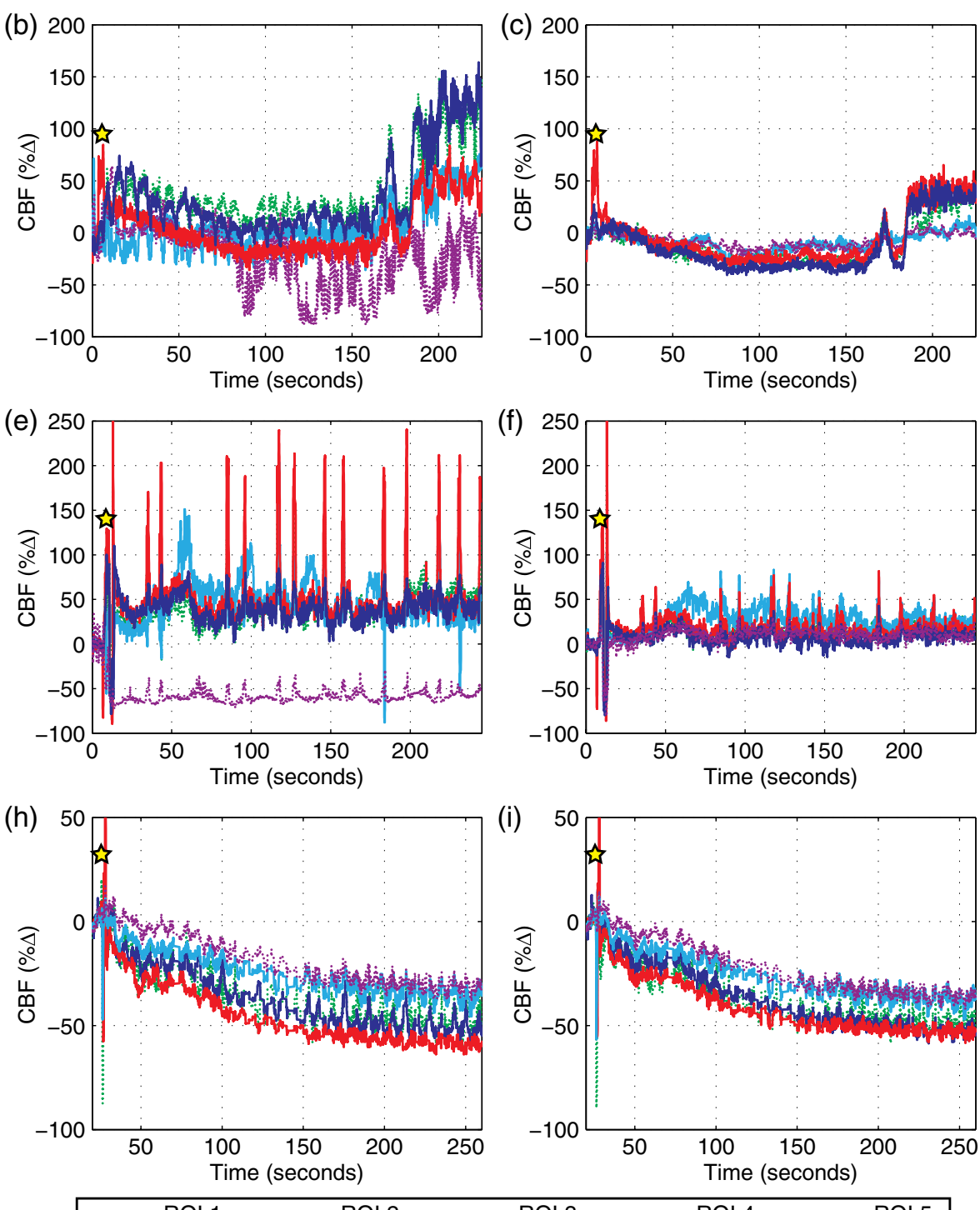

Fig. 7 A comparison between the time courses of ROls before and after translation-based image registration for three cases with cortical stimulation, patients 4,6 , and 10 for parts (a)-(c), (d)-(f), and (g)-(i), respectively. The images on the left $(a, d, g)$ display the speckle contrast image at the start of image acquisition averaged over 30 frames with a field of view of $\sim 2 \times 1.5 \mathrm{~cm}$ [same scale bar from Fig. 2(a)]. The color map displays high flows in red (low speckle contrast) and low flows in blue (high speckle contrast) with five numbered ROls overlaid in black where analysis is taking place. The dotted black oval indicates the location of stimulation in the field of view, and the yellow star indicates the time that the stimulation occurred. In (a) and (g), bipolar cautery was used with forceps close together (one oval). In (d), the Ojemann cortical stimulator was used, and the two ovals show the locations of both bipolar stimulators. The middle column of plots $(b, e, h)$ displays the time course of the original recorded CBF percent change $(\% \Delta)$ during the course of the intraoperative acquisition. The right column of plots (c, $\mathrm{f}$, i) displays the time course of the $\mathrm{CBF} \% \Delta$ after translation-based image registration of the image set. The CBF $\% \Delta$ is calculated using an average of $\sim 5 \mathrm{~s}$ of baseline flow before stimulation occurs. Plots for the same patient were plotted with the same axes for easier comparison before and after registration.

ranges from flat up to a $15-\mathrm{mm}$ radius sphere at the sulci. ${ }^{39}$ Because all images were recorded within the cortical gyri, where the osculating sphere radius should be much larger, we expect the remaining axial range of $3.1 \mathrm{~mm}$ after cardiac displacement to be sufficient for this study.

Other groups who have tested LSCI intraoperatively ${ }^{21-24}$ have not investigated the impact of motion compensation, which may be due to inherent differences in the instrumentation. Compared to the commercial LSCI system used in those studies, we integrated the LSCI hardware into the neurosurgical microscope. This had many benefits, including improving the surgical workflow, reducing the setup time for imaging, and increasing the surgeon's familiarity with the instrument. The relatively small FOV in our study made tissue motion compensation more critical, since smaller pixel resolution results in a larger relative tissue displacement in the images. Although not as sensitive to motion, large FOV images can also benefit from motion compensation. Another difference was the $4 \times$ faster frame rate 
(a)
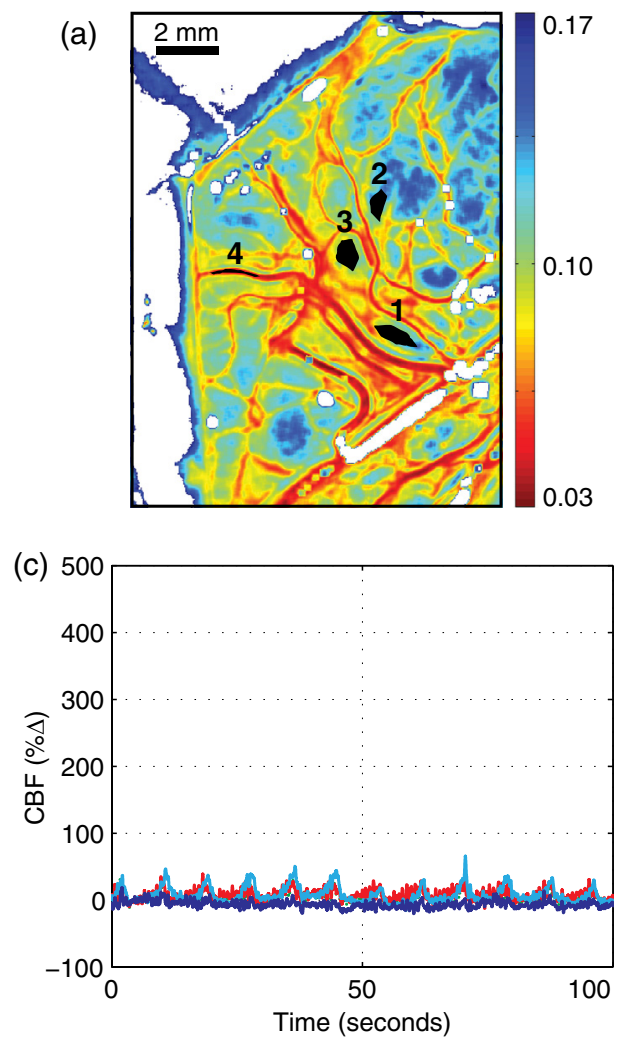

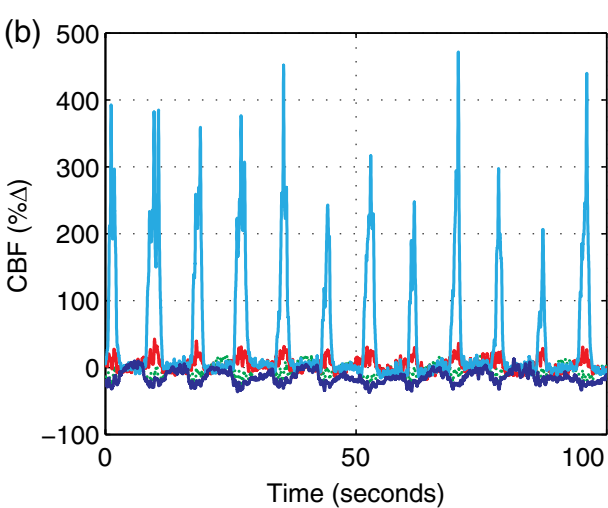

(d)

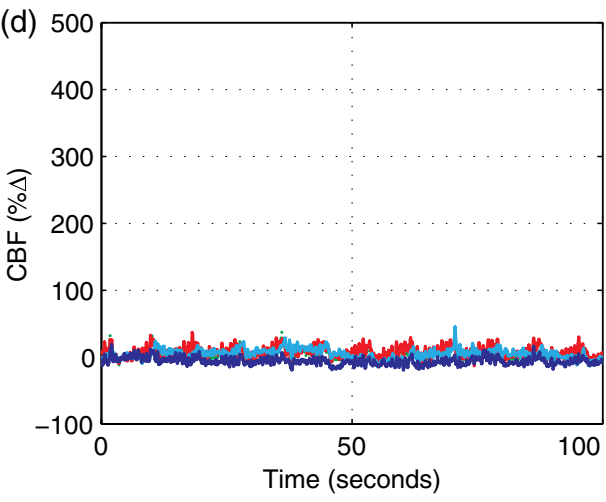

Fig. 8 A comparison between translation-based rigid registration and B-spline-based nonrigid registration illustrated with the time courses of ROls for one case, patient 5. The image in (a) shows the speckle contrast image at the start of image acquisition averaged over 30 frames with a cropped field of view of $\sim 1.6 \times 1.2 \mathrm{~cm}$ (scale bar included). The color map displays high flows in red (low speckle contrast) and low flows in blue (high speckle contrast) with four numbered ROls overlaid in black where analysis is taking place. (b) The original recorded CBF percent change $(\% \Delta)$ during the course of the intraoperative acquisition. (c) The time course of the $\mathrm{CBF} \% \Delta$ after translation-based image registration of the image set. (d) The time course of the CBF $\% \Delta$ after B-spline-based image registration of the image set. All plots are displayed with the same axes for easier comparison before and after registration and between the two registration methods.

of the camera used in this study. ${ }^{24}$ At rapid image acquisition, the camera more accurately captures the inherent physiological motion, which is why both cardiac and tissue motion correction significantly reduced CBF fluctuations in this study. The faster frame rate camera is beneficial because it allows more averaging of the acquired images, which improves the signal-to-noise ratio (SNR) in the image and ROI analysis. In this study, we

Table 2 Average and standard deviation of the percent difference between translation and B-spline registration over the time course of image acquisition. The regions correspond to the four numbered ROls shown in Figs. 4(a), 4(d), 4(g), and 8(a).

\begin{tabular}{lcccc} 
Region & $4(\mathrm{a})$ & $4(\mathrm{~d})$ & $4(\mathrm{~g})$ & $8(\mathrm{a})$ \\
\hline 1 & $0.58 \pm 0.44$ & $0.19 \pm 0.14$ & $0.59 \pm 0.42$ & $0.61 \pm 0.48$ \\
2 & $0.35 \pm 0.28$ & $0.76 \pm 0.51$ & $0.21 \pm 0.17$ & $0.66 \pm 0.48$ \\
3 & $1.6 \pm 1.2$ & $1.5 \pm 1.3$ & $0.32 \pm 0.29$ & $0.33 \pm 0.23$ \\
4 & $0.39 \pm 0.29$ & $2.9 \pm 2.6$ & $1.1 \pm 0.87$ & $7.0 \pm 5.1$ \\
\hline
\end{tabular}

performed averaging in the form of a moving average filter in postprocessing. For real-time display, five-frame averaging could be performed to improve the SNR, resulting in an effective frame rate of 20 images/second. As microscope-integrated LSCI becomes more widely adopted and as camera frame rates continue to increase, motion compensation will only increase in importance.

\section{Conclusion}

The results from this 10-patient pilot clinical study are promising and demonstrate that retrospective motion correction techniques can dramatically improve the accuracy for quantitatively monitoring relative blood flow over time. This is important for magnified imaging with high spatiotemporal resolution using a microscope-integrated LSCI system, and allows detection of blood flow changes after cortical stimulation. LSCI has the potential to be a valuable intraoperative monitoring tool for a variety of neurosurgical procedures, and should be further studied with real-time motion correction for possible addition to the current collection of available monitoring options. 


\section{Acknowledgments}

The authors would like to acknowledge the surgical staff at St. David's Hospital for their help during the experiments. The authors would also like to acknowledge Nazar Shaydyuk and Katherine Olin for their help with portions of the data analysis. This work was funded by grants from the Coulter Foundation, the National Institutes of Health (EB011556, NS082518, NS078791), the American Heart Association (14EIA18970041), and the Consortium Research Fellows Program.

\section{References}

1. D. L. Barrow, K. L. Boyer, and G. J. Joseph, "Intraoperative angiography in the management of neurovascular disorders," Neurosurgery 30(2), 153-159 (1992).

2. T. W. Vitaz et al., "Utility, safety, and accuracy of intraoperative angiography in the surgical treatment of aneurysms and arteriovenous malformations," AJNR Am. J. Neuroradiol. 20(8), 1457-1461 (1999).

3 . V. L. Chiang et al., "Routine intraoperative angiography during aneurysm surgery," J. Neurosurg. 96(6), 988-992 (2002).

4. J. R. Lopez, S. D. Chang, and G. K. Steinberg, "The use of electrophysiological monitoring in the intraoperative management of intracranial aneurysms," J. Neurol. Neurosur. Ps 66(2), 189-196 (1999).

5. H. Wiedemayer et al., "The impact of neurophysiological intraoperative monitoring on surgical decisions: a critical analysis of 423 cases," J. Neurosurg. 96(2), 255-262 (2002).

6. W. J. Loskota, "Intraoperative EEG monitoring," Semin. Anesth. Perioper. Med. Pain 24(4), 176-185 (2005).

7. N. Ogata et al., "Thermal diffusion blood flow monitoring during aneurysm surgery," Acta Neurochir. (Wien) 138(6), 726-731 (1996).

8. C. Thome et al., "Continuous monitoring of regional cerebral blood flow during temporary arterial occlusion in aneurysm surgery," J. Neurosurg. 95(3), 402-411 (2001).

9. Y. Kubo et al., "Revascularization and parent artery occlusion for giant internal carotid artery aneurysms in the intracavernous portion using intraoperative monitoring of cerebral hemodynamics," Neurosurgery 58(1), 43-49 (2006).

10. R. Stendel et al., "Intraoperative microvascular Doppler ultrasonography in cerebral aneurysm surgery," J. Neurol. Neurosurg. Psychiatry 68(1), 29-35 (2000).

11. E. Marchese et al., "Intraoperative microvascular Doppler in intracranial aneurysm surgery," Surg. Neurol. 63(4), 336-342 (2005).

12. E. Z. Kapsalaki et al., "The role of intraoperative micro-Doppler ultrasound in verifying proper clip placement in intracranial aneurysm surgery," J. Clin. Neurosci. 15(2), 153-157 (2008).

13. A. Raabe et al., "Prospective evaluation of surgical microscope-integrated intraoperative near-infrared indocyanine green videoangiography during aneurysm surgery," J. Neurosurg. 103(6), 982-989 (2005).

14. J. G. de Oliveira et al., "Assessment of flow in perforating arteries during intracranial aneurysm surgery using intraoperative near-infrared indocyanine green videoangiography," Neurosurgery 62(6 Suppl. 3), 1300-1310 (2007).

15. R. Dashti et al., "Microscope-integrated near-infrared indocyanine green videoangiography during surgery of intracranial aneurysms: the Helsinki experience," Surg. Neurol. 71(5), 543-550 (2009).

16. V. G. Khurana, K. Seow, and D. Duke, "Intuitiveness, quality and utility of intraoperative fluorescence videoangiography: Australian neurosurgical experience," Br. J. Neurosurg. 24(2), 163-172 (2010).

17. A. Gruber et al., "Prospective comparison of intraoperative vascular monitoring technologies during cerebral aneurysm surgery," Neurosurgery 68(3), 657-673 (2011).

18. S. Bacigaluppi et al., "Monitoring techniques for prevention of procedurerelated ischemic damage in aneurysm surgery," World Neurosurg. 78(3-4), 276-288 (2012).

19. J. D. Briers, "Laser Doppler, speckle and related techniques for blood perfusion mapping and imaging," Physiol. Meas. 22(4), R35-R66 (2001).

20. D. A. Boas and A. K. Dunn, "Laser speckle contrast imaging in biomedical optics," J. Biomed. Opt. 15(1), 011109 (2010).

21. N. Hecht et al., "Intraoperative monitoring of cerebral blood flow by laser speckle contrast analysis," Neurosurg. Focus 27(4), E11 (2009).
22. N. Hecht et al., "Laser speckle imaging allows real-time intraoperative blood flow assessment during neurosurgical procedures," J. Cereb. Blood Flow Metab. 33(7), 1000-1007 (2013).

23. E. Klijn et al., "Laser speckle imaging identification of increases in cortical microcirculatory blood flow induced by motor activity during awake craniotomy," J. Neurosurg. 118(2), 280-286 (2013).

24. J. Woitzik et al., "Propagation of cortical spreading depolarization in the human cortex after malignant stroke," Neurology 80(12), 1095-1102 (2013).

25. "American National Standard for the Safe Use of Lasers," in Proc. ANSI Z136.1-2007, Laser Institute of America, Orlando, FL (2007).

26. J. D. Briers and S. Webster, "Laser speckle contrast analysis (LASCA): a nonscanning, full-field technique for monitoring capillary blood flow," J. Biomed. Opt. 1(2), 174-179 (1996).

27. W. J. Tom, A. Ponticorvo, and A. K. Dunn, "Efficient processing of laser speckle contrast images," IEEE Trans. Med. Imag. 27(12), 1728-1738 (2008)

28. R. Bandyopadhyay et al., "Speckle-visibility spectroscopy: a tool to study time-varying dynamics," Rev. Sci. Instrum. 76(9), 093110 (2005).

29. A. K. Dunn et al., "Dynamic imaging of cerebral blood flow using laser speckle," J. Cereb. Blood Flow Metab. 21(3), 195-201 (2001).

30. C. Ayata et al., "Laser speckle flowmetry for the study of cerebrovascular physiology in normal and ischemic mouse cortex," J. Cereb. Blood Flow Metab. 24(7), 744-755 (2004).

31. A. B. Parthasarathy et al., "Laser speckle contrast imaging of cerebral blood flow in humans during neurosurgery: a pilot clinical study," J. Biomed. Opt. 15(6), 066030 (2010).

32. L. M. Richards et al., "Intraoperative laser speckle contrast imaging for monitoring cerebral blood flow: results from a 10-patient pilot study," Proc. SPIE 8207, 82074L (2012).

33. L. M. Richards et al., "Laser speckle imaging of cerebral blood flow," in Optical Methods and Instrumentation in Brain Imaging and Therapy, S. J. Madsen, Ed., pp. 117-136, Springer, New York (2013).

34. S. Klein et al., "Elastix: a toolbox for intensity-based medical image registration," IEEE Trans. Med. Imag. 29(1), 196-205 (2010).

35. A. Raabe et al., "Laser Doppler imaging for intraoperative human brain mapping," Neuroimage 44(4), 1284-1289 (2009).

36. P. Miao et al., "High resolution cerebral blood flow imaging by registered laser speckle contrast analysis," IEEE Trans. Biomed. Eng. 57(5), 1152-1157 (2010).

37. H. Sun et al., "Cortical surface tracking using a stereoscopic operating microscope," Neurosurgery 56([ONS Suppl]1), ONS-86-ONS-97 (2005).

38. L. D. Griffin, "The intrinsic geometry of the cerebral cortex," J. Theor. Biol. 166(3), 261-273 (1994).

39. L. Palaniyappan et al., "Folding in the prefrontal cortex in schizophrenia: regional differences in gyrification," Biol. Psychiatry 69(10), 974 979 (2011).

Lisa M. Richards received her BSE degree from Duke University in 2008 and her MSE degree from the University of Texas at Austin in 2012, both in biomedical engineering. Currently, Lisa is a doctoral candidate working in the Functional Optical Imaging Laboratory in the Department of Biomedical Engineering at the University of Texas at Austin.

Erica L. Towle received her BS degree in electrical engineering from the University of Wyoming in 2008. She received her MSE degree in 2010 and her PhD in 2012 from the University of Texas at Austin, both in biomedical engineering. After graduate school, Erica worked briefly as a National Research Council postdoctoral fellow with the Air Force Research Laboratory in San Antonio, Texas. Currently, Erica works on innovative hardware development at Microsoft.

Douglas J. Fox, Jr. is a neurosurgeon at St. David's Hospital, executive medical director of the NeuroTexas Institute, and adjunct professor of biomedical engineering at the University of Texas at Austin. His clinical interests include cerebrovascular neurosurgery, skull base surgery, and complex spinal surgery. His research interests include the application of novel intraoperative imaging methods for neurosurgery.

Andrew K. Dunn is an associate professor of biomedical engineering and a fellow of the Herring Professorship at the University of Texas at Austin. His research interest is in the field of optical microscopy for investigation of brain function, particularly in stroke and neurovascular disorders. 